\title{
Accelerated Chemical Aging of Crystalline Nuclear Waste Forms: A Density Functional Theory Study of ${ }^{109} \mathrm{Cd}_{x}{ }^{109} \mathrm{Ag}_{1-x} \mathrm{~S}$
}

\author{
B. Dorado ${ }^{\mathrm{a}, \mathrm{b}}$, B.P. Uberuaga ${ }^{\mathrm{b}}$, N.A. Marks ${ }^{\mathrm{c}}$, C.R. Stanek $\mathrm{U}^{\mathrm{b}, *}$ \\ ${ }^{a}$ CEA, DAM, DIF, F-91297 Arpajon, France \\ ${ }^{b}$ Materials Science and Technology Division, Los Alamos National Laboratory, Los Alamos, \\ NM 87545, United States \\ ${ }^{c}$ Discipline of Physics and Astronomy, Curtin University, GPO Box U1987, Perth, WA \\ 6845, Australia
}

\begin{abstract}
Recently, a combined experimental-theoretical approach to assess the effect of daughter product formation on the stability of crystalline compounds comprised of radioisotopes has been developed. This methodology was motivated by the potential impact on crystalline nuclear waste form stability of a significant fraction of the constituent atoms undergoing transmutation. What is particularly novel about this approach is the experimental use of very short-lived isotopes to accelerate the chemical evolution that occurs during decay. In this paper, we present results of density functional theory (DFT) calculations that have been performed in support of corresponding experiments on the ${ }^{109} \mathrm{Cd}_{x}{ }^{109} \mathrm{Ag}_{1-x} \mathrm{~S}$ material system. ${ }^{109} \mathrm{Cd}$ has been selected in order to simulate the decay of important "short-lived" fission products ${ }^{137} \mathrm{Cs}$ or ${ }^{90} \mathrm{Sr}$ (which decay via $\beta$ - to ${ }^{137} \mathrm{Ba}$ and ${ }^{90} \mathrm{Zr}$ respectively with $\approx 30$-year half-lives). By comparison, ${ }^{109} \mathrm{Cd}$ decays by electron capture with a half-life of 109 days to ${ }^{109} \mathrm{Ag}$. DFT results predict the formation of heretofore unobserved $\mathrm{Cd}_{x} \mathrm{Ag}_{1-x} \mathrm{~S}$ structures, which support corresponding experiments and ultimately may have implications for waste form stability.
\end{abstract}

Keywords: cadmium sulfide, silver sulfide, nuclear waste forms,

\footnotetext{
* Corresponding author

Email address: stanek@lanl.gov (C.R. Stanek)
}

Preprint submitted to NIM B

October 23, 2014 
transmutation, density functional theory

\section{Introduction}

The robust encapsulation of nuclear waste is critical to realizing a closed fuel cycle. For many years, crystalline waste forms have been considered as candidates for waste host phases as an alternative to glass. Although the focus on 5 candidate waste form stability has centered on the $\alpha$-decay of actinides, there is also significant motivation to consider $\beta$-decay isotopes. For used nuclear fuel, it has been shown that significant volumetric increases in geological repositories are possible if actinides (major source of radiotoxicity) and short-lived Cs and $\mathrm{Sr}$ (which are responsible for a considerable fraction of the heat load) are removed from the waste stream [1]. There is also a significant amount of military waste that exists from the production and subsequent reduction of nuclear weapons. From this military waste, in addition to $\approx 50$ metric tons of surplus $\mathrm{Pu}\left(\right.$ mostly $\left.{ }^{239} \mathrm{Pu}\right)$ 2, there are $\approx 2,000$ capsules containing ${ }^{137} \mathrm{CsCl}$ and ${ }^{90} \mathrm{SrF}_{2}$ that accounts for $\approx 60$ million curies of radioactivity and have been described as the most lethal single source of radiation in the United States other than inside an operating reactor [3]. For actinides, an important concern is the radiation damage incurred in a crystalline waste form due to $\alpha$-decay events leading to the potential for amorphization and subsequent modification of materials properties that govern waste form performance [4, 5, 6, 7, 8. However, for the short-lived isotopes of $\mathrm{Sr}$ and $\mathrm{Cs}$, radiation damage is less of a concern than is the chemical evolution that occurs during $\beta$-decay. For example, ${ }^{137} \mathrm{Cs}$ and ${ }^{90} \mathrm{Sr}$ decay with half-lives $\approx 30$ years to chemically different daughter products ${ }^{137} \mathrm{Ba}$ and ${ }^{90} \mathrm{Zr}$, respectively. These decay processes are accompanied by relatively small recoil energies, leading to little generation of damage [9]. However, given the 25 large change in valence state and ionic radius, the stability of crystalline waste forms comprised of ${ }^{137} \mathrm{Cs}$ or ${ }^{90} \mathrm{Sr}$ is likely to be affected by daughter product formation [10, 11]. Unfortunately, given the $\approx 30$-year half life of ${ }^{137} \mathrm{Cs}$ and ${ }^{90} \mathrm{Sr}$, experiments to assess the stability of candidate waste forms would require 
several hundred years.

In order to overcome the temporal barrier that prevents assessment of the impact of daughter product formation on waste form stability, we have recently developed a so-called "accelerated chemical aging" approach [12. In this approach, we complement experiments using very short-lived isotopes to mimic the chemical evolution that would occur in an actual waste form with DFT calculations. We have commenced experiments involving compounds comprised of ${ }^{109} \mathrm{Cd}$, which decays via electron capture to ${ }^{109} \mathrm{Ag}$ with a half-life of 462 days. ${ }^{109} \mathrm{Cd}$ satisfies all criteria specified in our original definition of accelerated chemical aging 12 in that the half-life is conducive to experimentation, the daughter product is chemically distinct, the sample would exhibit sufficiently ${ }_{40}$ low radioactivity, and the isotope is available. For these experiments, the ${ }^{109} \mathrm{Cd}$ radionuclide was produced via proton irradiation of an In target in the 100 $\mathrm{MeV}$ proton beam at the Isotope Production Facility located at the Los Alamos Neutron Science Center. ${ }^{109} \mathrm{CdS}$ samples were subsequently produced for examination by X-ray diffraction and electron microscopy. It is worth noting that the 45462 day half-life of ${ }^{109} \mathrm{Cd}$ allows for experiments that occur over several years rather than several centuries if performed on ${ }^{137} \mathrm{Cs}$ or ${ }^{90} \mathrm{Sr}$. While ${ }^{109} \mathrm{Cd}$ is not a fission product of interest, nor is CdS proposed as a candidate waste form, the system is attractive as it enables us to explore the possible consequences of in situ daughter product formation via the relative ease of forming a sulfide compound in a hot cell environment.

The results of the experimental studies will be presented in a future paper. In this paper, we present results of DFT calculations that simulate time evolution by varying the composition of the ${ }^{109} \mathrm{Cd}_{x}{ }^{109} \mathrm{Ag}_{1-x} \mathrm{~S}$ system, where higher concentrations of $\mathrm{Ag}$ represent further in the future during the decay of ${ }^{109} \mathrm{Cd}$. ${ }_{55} \mathrm{CdS}$ crystallizes in the hexagonal wurtzite structure, space group $\mathrm{P} 6{ }_{3} \mathrm{mc}$ (No. 186) [13, although it is also found in the cubic sphalerite (zincblende) structure. There is a structural relationship between wurtzite and sphalerite as has been discussed in detail previously [14, 15]. CdS is a wide band gap semiconductor a commonly used pigment, but has received a great deal of research attention 
for potential electronic applications [16, 17, 18. Under high pressure, CdS has been observed to transform to the rocksalt structure [19, 20]. In addition to wurtzite/zincblende and rocksalt (given the observation of it at high pressure), we have also considered two other structures that preserve 1:1 Me (i.e. Cd or $\mathrm{Ag})$ to S stoichiometry. First, a previous DFT study on high pressure phases of ${ }_{65} \mathrm{MgO}$ and $\mathrm{GaN}$ from wurtzite to rocksalt identified an intermediate structure, which was referred to as h-MgO 21]. This structure, which can be envisioned as a relaxed wurtzite structure, allows for a smooth transition between rocksalt and wurtzite. Similarly, we have considered a body centered tetragonal (BCT) structure, which is a high temperature structure of $\beta$ - $\mathrm{BeO}$ (space group $\left.{ }_{70} \mathrm{P} 4_{2} / \mathrm{mnm}\right)$, whereas the low temperature structure of $\alpha$-BeO is wurtzite [22]. The goal of this study is to determine the thermodynamic ordering of these four structures at a variety of $\mathrm{Ag}$ concentrations, which correspond to different instances in the decay process, in order to provide some theoretical insight into the possible evolution of the experimental sample, see Figure 1. These structures have been shown to be relevant for other compositions at either high temperature or pressure. The reader is referred elsewhere for a detailed analysis of the crystallographic relationships [14, 15, 21, 22.

\section{Methodology}

All DFT calculations were performed using the ABINIT package [23, 24]. The Kohn-Sham wave functions were calculated within the projector augmentedwave (PAW) formalism [25], in the generalized gradient approximation (GGA) of the DFT. The cutoff energy was set to $800 \mathrm{eV}$ and the $k$-point meshes used in the calculations depend on the structures considered, namely wurtzite (WZ), rocksalt ( $\mathrm{RS})$, sphalerite (SP), $h$-MgO and body-centered tetragonal (BCT).

${ }_{85}$ In addition to calculating thermodynamic and structural properties of these crystallographically different compounds (hence different $k$-point meshes), DFT was also employed to assess variations of electronic structure as a function of Ag concentration, which consequently requires finer $k$-point meshes. For all 


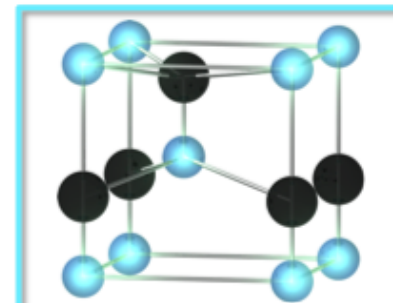

wurtzite

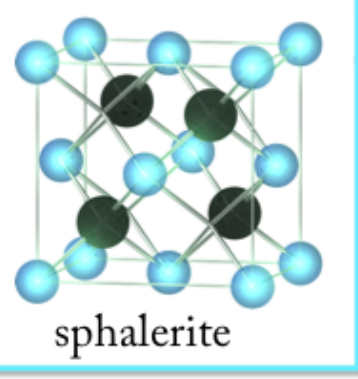

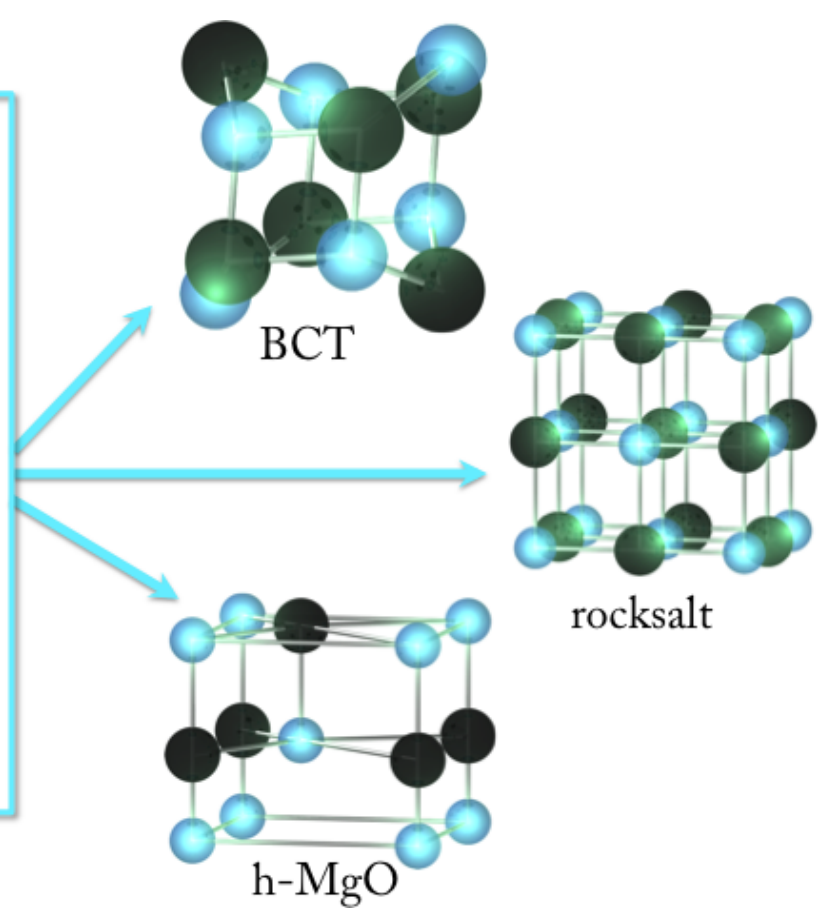

Figure 1: Crystal structures considered during the chemical evolution of $\mathrm{Cd}_{1-x} \operatorname{Ag}_{x} \mathrm{~S}$, where luminescent green atoms correspond to radioisotopes, e.g. ${ }^{109} \mathrm{Cd}$, and dark atoms correspond to stable isotopes, i.e. S. At $\mathrm{t}=0$, wurtzite and sphalerite are the ground state structures of $\mathrm{Cd}_{1-x} \mathrm{Ag}_{x} \mathrm{~S}$. BCT refers to the body-centered tetragonal structure. DFT calculations show how the stability of these structures is impacted by the increase of Ag concentration.

structures and compositions considered, convergence with respect to the cutoff energy and the $k$-point mesh was ensured to be lower than $1 \mathrm{meV} /$ atom.

There are two methods that are typically employed to model alloys: the Korringa-Kohn-Rostoker (KKR) method [26, 27] and the special quasirandom structures (SQS) approach [28. In this work, we use a 32-atom SQS to model all $\mathrm{Cd}_{1-x} \mathrm{Ag}_{x} \mathrm{~S}$ compounds, with $x=0.06,0.25,0.50$, and 0.75 . We performed 95 two sets of calculations: in the first set, we took into account all possible symmetries in all structures (WZ, RS, SP, BCT, and $h$-MgO) and we kept the volume constant and equal to that corresponding to the structure with $0 \%$ silver. However, in the second set, we released all symmetry constraints and allowed for a full relaxation of both volume and atomic positions. The first set is useful 
the silver concentration. The constant volume calculations also provide a useful reference for understanding how the ideal crystalline stability depends on $\mathrm{Ag}$ concentration.

\section{Results}

because it allows investigation of the pressure effects caused by the increase in Thermodynamic Stability. Figure 2 describes DFT results of $\mathrm{Cd}_{1-x} \mathrm{Ag}_{x} \mathrm{~S}$ thermodynamic stability, normalized to the energy of the wurtzite structure at each value of x considered, and Figure 2(a) and 2(b) correspond to the constrained and unconstrained case, respectively. As previously mentioned, at $0 \%$ silver, i.e. $\mathrm{x}=0$ (and $\mathrm{t}=0)$, CdS crystallizes in the WZ structure, which is correctly predicted to be the most stable structure at that composition (for calculations with and without symmetry). At $\mathrm{x}=0$, by order of decreasing stability, we find $\mathrm{WZ} \approx \mathrm{SP}>h-\mathrm{MgO}>\mathrm{BCT}>\mathrm{RS}$. Although expected based on their crystallographic similarity, it is worth noting that the energies for SP are very close to WZ for all concentrations of silver considered.

It is evident in Figure 2 that the results of constrained and unconstrained calculations (i.e. Figure $2 \mathrm{a}$ and $2 \mathrm{~b}$ ) yield similar qualitative trends of energetic ordering. Notably, the wurtzite and sphalerite structures are preferred from $\mathrm{x}$ $=0$ to $\mathrm{x} \approx .5$ (or $50 \% \mathrm{Ag})$. A very slight preference for sphalerite over wurtzite develops at $\mathrm{x} \approx 0.125$ and is maintained until $\mathrm{x}=1$. For both constrained and unconstrained cases, rocksalt is the lowest energy phase at $\mathrm{x}=1$, followed by $h$ $\mathrm{MgO}$. The main difference between the constrained and unconstrained datasets occurs in the intermediate compositions. For example, for the unconstrained calculations the $h$-MgO phase may be favored over rocksalt between $\mathrm{x}=0.5$ and $\mathrm{x}=0.75$. A closer examination of the $h-\mathrm{MgO}$ results suggest that this phase is rather sensitive to compositional variation. For instance, at $6 \%$ and $75 \%$ silver, we can see that the $h$-MgO phase transforms into the WZ and RS phases, respectively, when the volume constraint is relaxed.

From the constrained calculations, we are able to calculate that $25 \%$ silver 


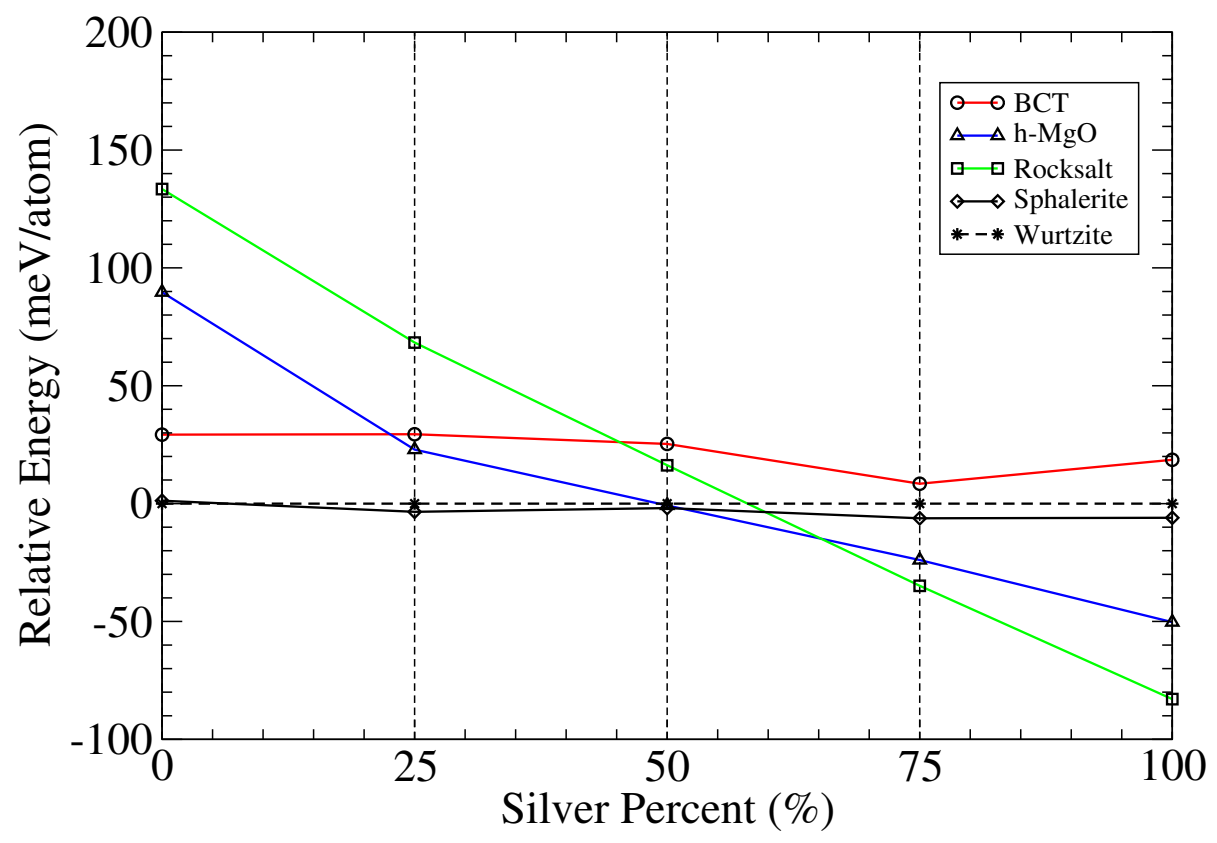

(a) Constrained

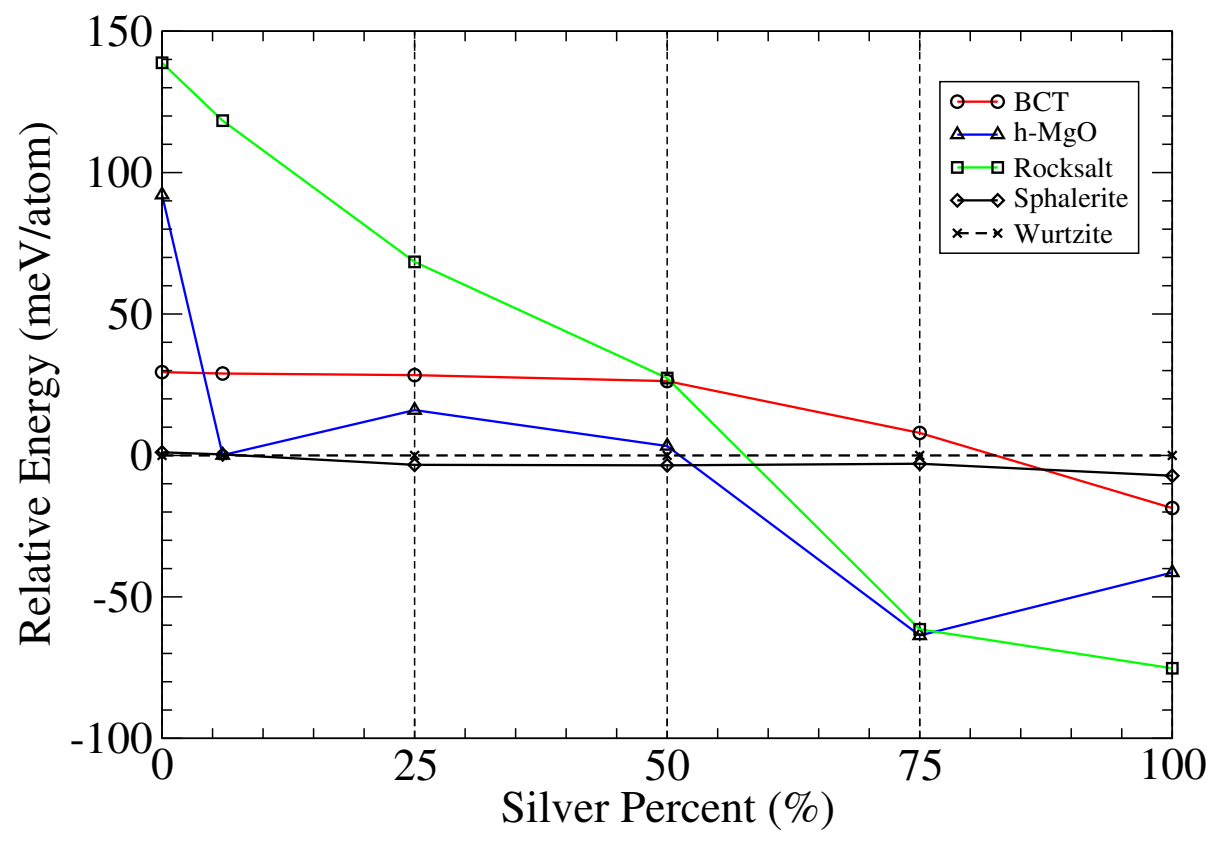

(b) Unconstrained

Figure 2: Thermodynamic stabilities (relative to the wurtzite phase) of all phases with respect to increasing silver percent for the two types of calculations: constrained (a) and unconstrained (b). 


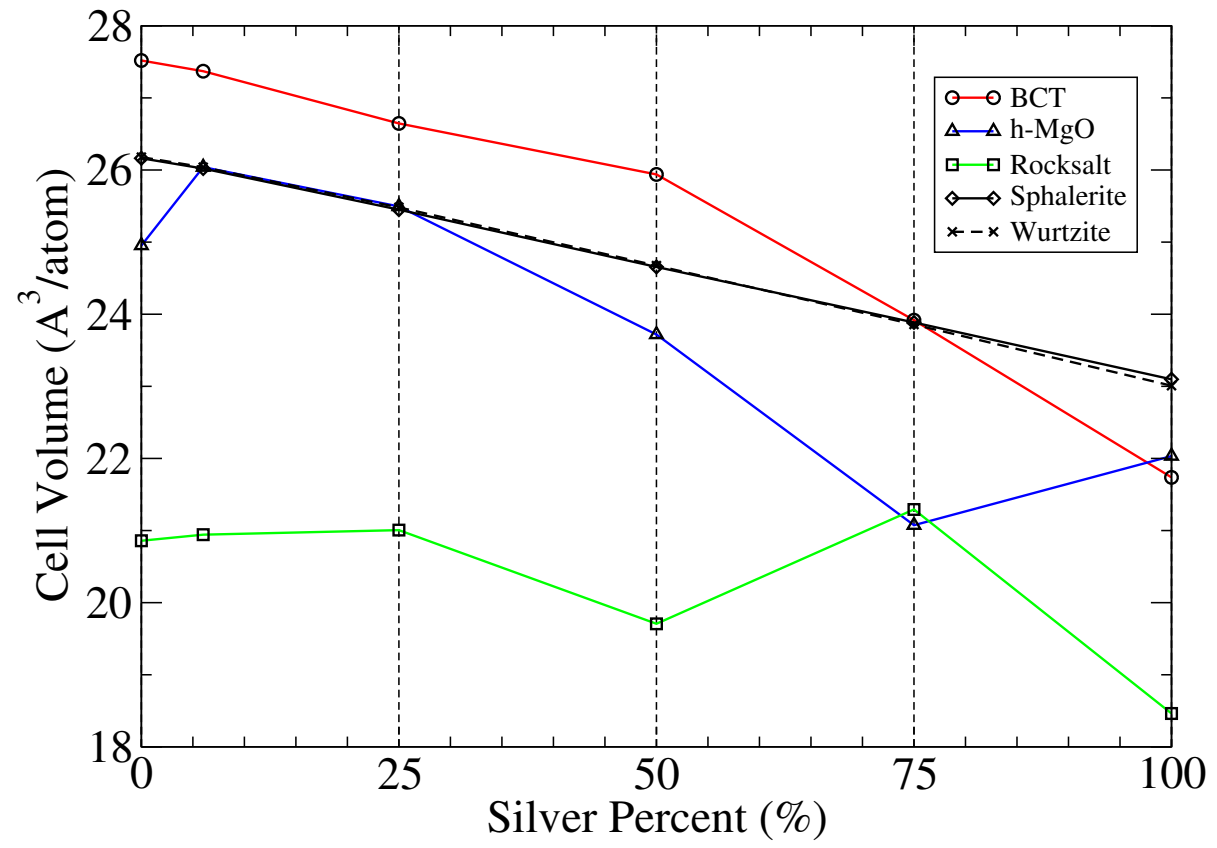

Figure 3: Evolution of cell volumes (in $\AA^{3} /$ atom) of all $\mathrm{Cd}_{1-x} \mathrm{Ag}_{x} \mathrm{~S}$ structures with respect to silver content. 
corresponds to a pressure of $-1.143 \mathrm{GPa}$ and $50 \%$ silver to $-2.352 \mathrm{GPa}$. The observed trend is that by adding silver, a pressure effect is chemically induced and the material tends to contract. Figure 3 shows the evolution of the cell volume (in $\AA^{3} /$ atom) with respect to the silver content. We see from Figure 3 that cell volume decreases with increasing Ag concentration. Interestingly, this result is similar to the decrease in cell volume observed in the $\mathrm{Cs}_{1-x} \mathrm{Ba}_{x} \mathrm{Cl}$ system [29], which decays by $\beta$ - rather than electron capture. This suggests that the formation of daughter products sufficiently alters the electronic structure such that basic ionic radius arguments may not be valid, since both electron capture and emission may lead to daughter products that contract the material. Further work is required to determine if daughter product formation always results in the equivalent of an applied pressure, no matter the decay mechanism. The concentration of silver at which the WZ phase becomes relatively unstable (i.e. $50 \%$ ) corresponds to an applied pressure of $\approx 2.3 \mathrm{GPa}$. At $65 \%$ silver, the RS phase is more stable, which corresponds to an applied pressure of $\approx 2.7 \mathrm{GPa}$. Note that these pressures are very similar to the pressures at which a WZ to RS phase transition has been observed experimentally ( $\approx 2 \mathrm{GPa}$, see e.g. [19]). Therefore, the DFT calculations seem to indicate that with increasing silver percent in $\mathrm{CdS}$, pressure effects will induce a phase transformation from WZ to $\mathrm{RS}$ with the $h$ - $\mathrm{MgO}$ phase as a possible intermediate metastable state. These results also suggest that applied pressure experiments may result in similar behavior as transmutation-induced chemical strain. Further, such experiments are, in practice, significantly simpler to perform than the accelerated chemical aging experiments described here. Thus, they may prove a suitable complement to, if not an explicit surrogate for, direct aging studies.

In addition to the relative stability of possible $\mathrm{Cd}_{1-x} \mathrm{Ag}_{x} \mathrm{~S}$ phases, it is also important to understand the propensity for phase separation, as the real samples may experience enough decay heat to allow for mass transport. For the $\mathrm{Cd}_{1-x} \mathrm{Ag}_{x} \mathrm{~S}$ system, this can be expressed as:

$$
\mathrm{Cd}_{1-x} \mathrm{Ag}_{x} \mathrm{~S} \rightleftharpoons(1-x) \mathrm{CdS}+x \mathrm{Ag}+x \mathrm{~S}
$$


where $\mathrm{CdS}$ is cadmium sulfide in the WZ phase, $\mathrm{Ag}$ is face-centered cubic $\mathrm{Ag}$ metal, and $\mathrm{S}$ is elemental sulphur (128-atom unit cell).

Figure 4 shows the results of calculations for both sets of thermodynamic stabilities of all phases with respect to increasing silver concentration, and plotted relative to phase decomposition. From Figure 4 we can draw the same conclusions as from Figure 2 At $0 \%$ silver, we find CdS to be the most stable in the WZ structure, then $\mathrm{SP}>h-\mathrm{MgO}>\mathrm{BCT}>\mathrm{RS}$. We also see that the SP phase is very near in energy to the WZ phase, for all compositions. Overall, it is observed that the WZ/SP phase is the most stable phase up to $50 \%$ silver, at which point there is a very small range of stability of the $h-\mathrm{MgO}$ phase that cannot be exactly determined because of the phase transformation to $h-\mathrm{MgO} \rightarrow \mathrm{RS}$ at $75 \%$ silver. Eventually, the RS structure becomes more stable. Here again, the DFT calculations seem to indicate that a phase transition from WZ-CdS to RS-AgS may occur with the $h-\mathrm{MgO}$ structure serving as a possible intermediary phase. We also note that no matter the intermediate phase, it is higher in energy than either of the end members. At some concentration of Ag, the thermodynamic stability begins to increase. Finally, it is worth noting that all phases are unstable with respect to phase decomposition into WZ/SP-CdS, Ag, and elemental S. This result supports our initial experimental observations, where the results suggest that the $\mathrm{Cd}_{1-x} \mathrm{Ag}_{x} \mathrm{~S}$ sample may contain several different phases, including WZ/SP-CdS. Initial experimental results suggest that the $\mathrm{Cd}_{1-x} \mathrm{Ag}_{x} \mathrm{~S}$ sample may contain several different phases, including: WZ/SP-CdS, WZ-Cd ${ }_{1-x} \mathrm{Ag}_{x} \mathrm{~S}$, nanoparticle silver and at least two yet unidentified phases that very well may be the $\mathrm{BCT}$ or $h-\mathrm{MgO}$ structures.

Finally, we have considered contributions to thermodynamic stability from configurational entropy, $\mathrm{S}_{\text {conf }}$, where

$$
S_{\text {conf }}=k_{B} * \ln \left(\frac{n_{\text {sites }} !}{\left(n_{\text {sites }}-n_{\text {silver }}\right) ! * n_{\text {silver }} !}\right) .
$$

The results of calculations of thermodynamic stability including configurational 185 entropy are provided in Figure 5. The general trend of phase evolution observed 


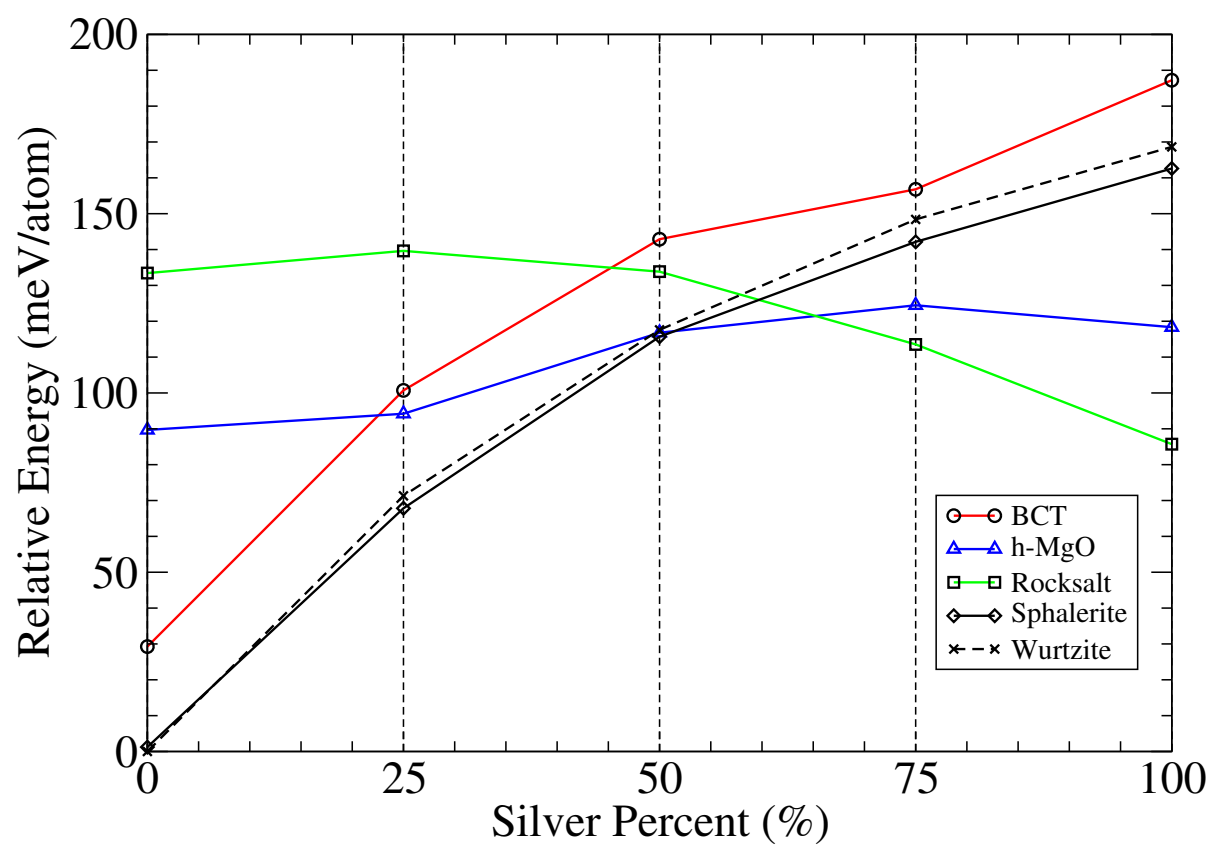

(a) Constrained

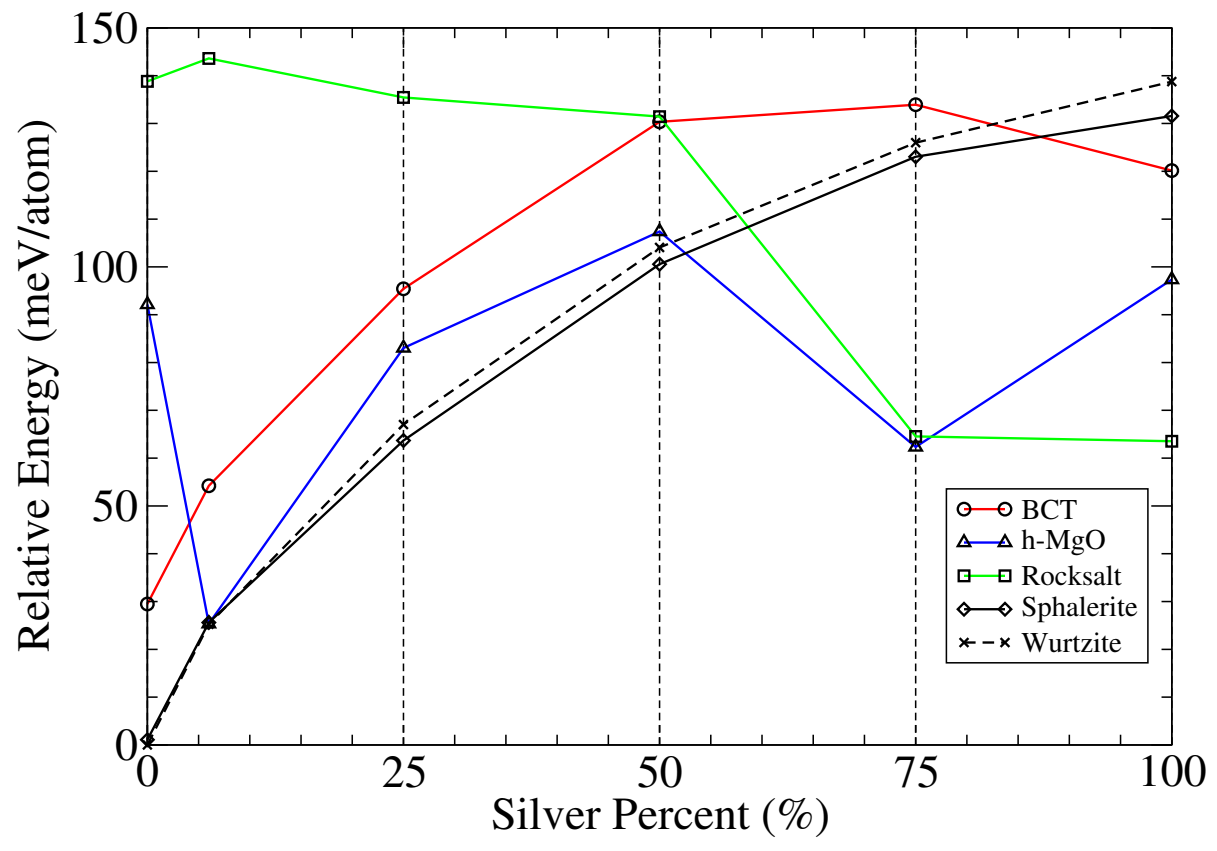

(b) Unconstrained

Figure 4: Thermodynamic stabilities (relative to phase decomposition) of all phases with respect to increasing silver percent for the two types of calculations: constrained (top) and unconstrained (bottom). 


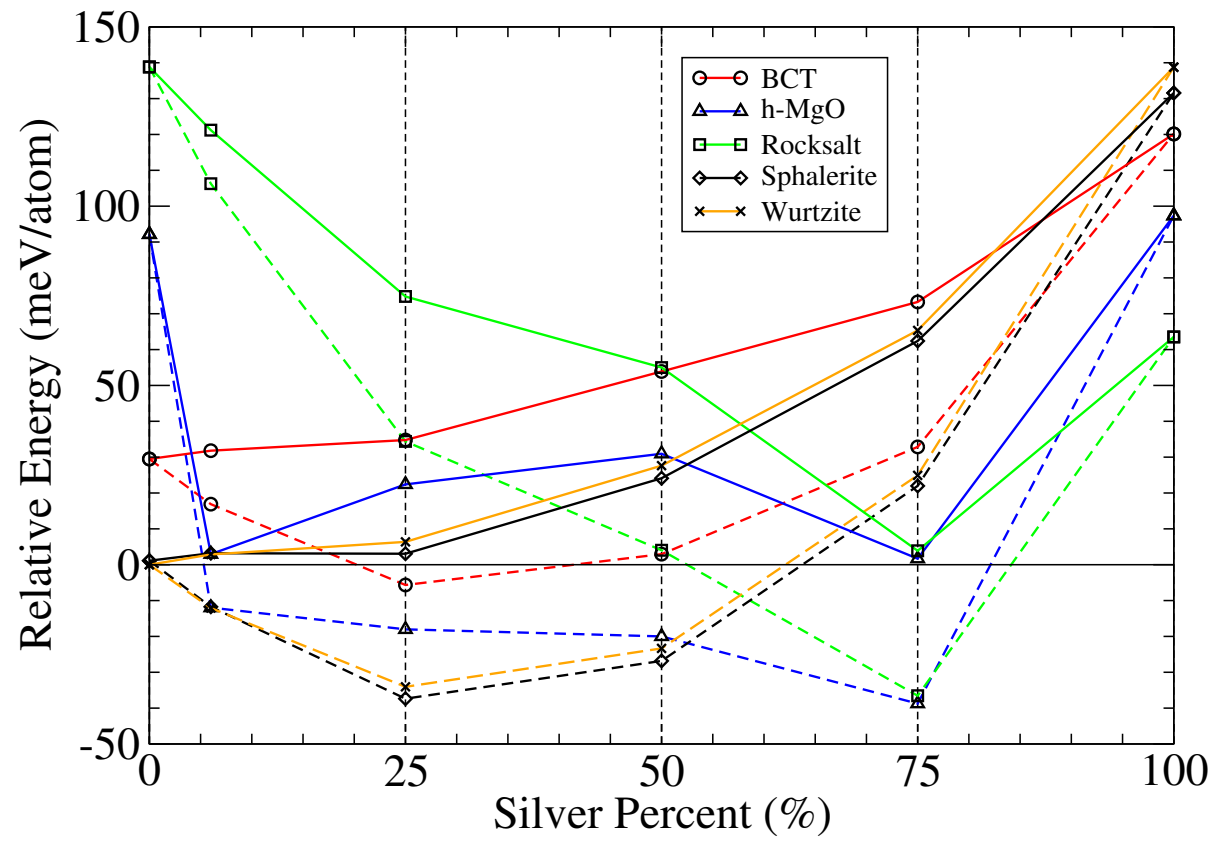

Figure 5: Thermodynamic stabilities (relative to phase decomposition) of unconstrained structures, including the configurational entropy, at two temperatures: $300 \mathrm{~K}$ (plain lines) and 500K (dashed lines). 
in Figure 5 is similar to what was observed without including configurational entropy. For compositions with silver concentration less than $\approx 50 \%$, the preferred structures are wurtzite and sphalerite. For concentrations above $\approx 50 \%$ silver, the preference for wurtzite and sphalerite is replaced by h-MgO and rocksalt. However, a noticeable difference when including configurational entropy is that all structures appear more stable with respect to dissociation. In fact, at (500 K, denoted by dashed trend lines in Figure 5), there are structures that are more stable than dissociation. To fully understand the role of entropy on thermodynamic stability of these structures requires further study. Implications of phase evolution. As mentioned in the Introduction, the stability of crystalline waste forms comprised of ${ }^{137} \mathrm{Cs}$ or ${ }^{90} \mathrm{Sr}$ is likely to be affected by daughter product formation. In this subsection, we use DFT calculations to provide some clues about how the compositional evolution may impact waste form stability. First, and as has been discussed previously [29], the volume change associated with daughter product formation is a point of concern. Significant volume change may lead to cracking and increased surface area and reactivity. Therefore, candidate waste forms that undergo minimal volume change are preferred. From Figure 3 , it is clear that a significant volume change is predicted to occur during the decay of ${ }^{109} \mathrm{Cd}$ to ${ }^{109} \mathrm{Ag}$.

\begin{tabular}{ccc} 
& WZ-CdS & RS-AgS \\
\hline $\mathrm{C}_{11}$ & $75.6(86.5)$ & 139.4 \\
$\mathrm{C}_{12}$ & $43.9(54.0)$ & 51.7 \\
$\mathrm{C}_{44}$ & $14.4(15.0)$ & 15.3 \\
$\mathrm{C}_{13}$ & $43.4(47.0)$ & \\
$\mathrm{C}_{33}$ & $92.2(96.5)$ & \\
$\mathrm{E}_{\text {coh }}$ & 4.8 & 5.2
\end{tabular}

Table 1: Elastic constants (in GPa) and cohesive energy (in eV) of WZ-CdS and RS-AgS, compared to experimental values in parentheses where available 31 . 


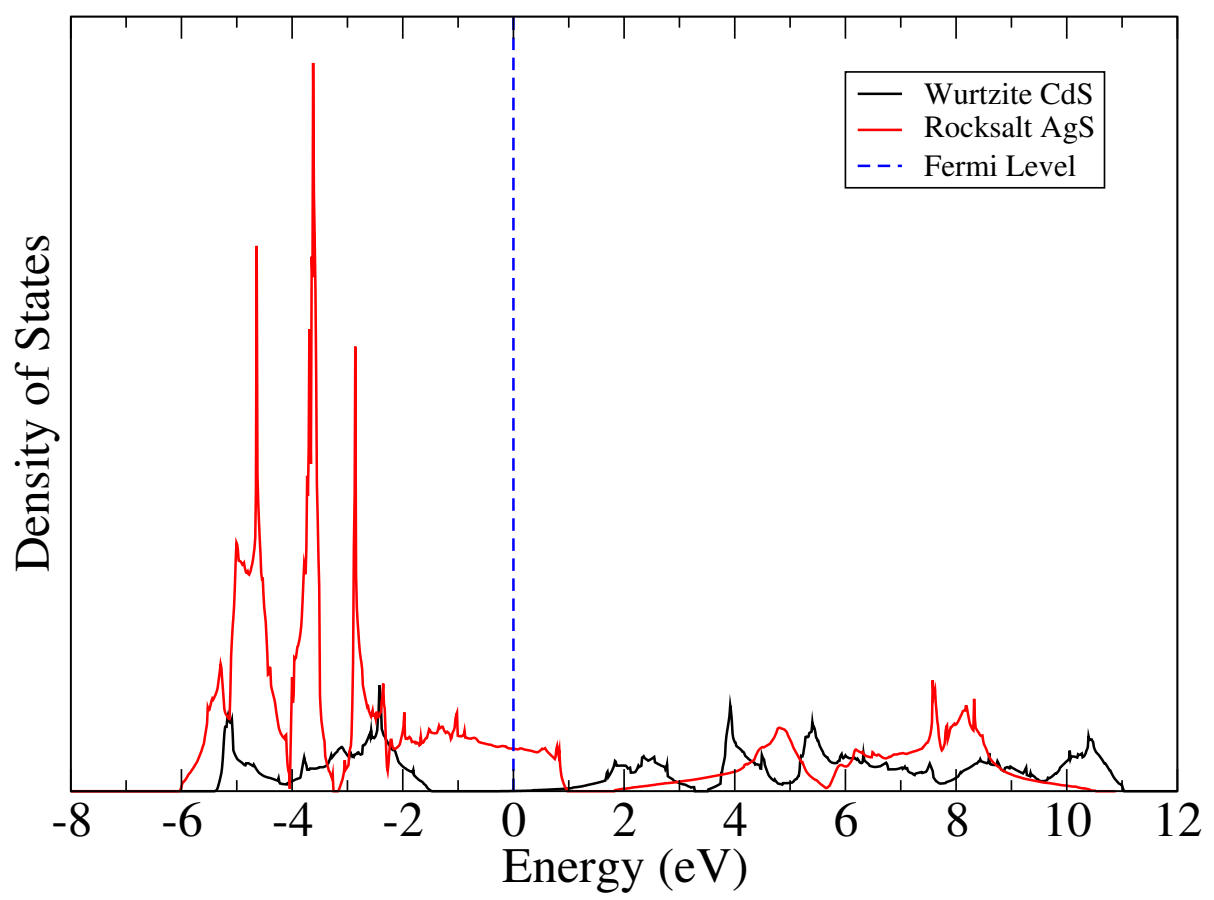

Figure 6: Electron density of states of WZ-CdS and RS-AgS.

formation that provide insights into waste form performance. For example, Table 1 shows the elastic constants (in GPa) and cohesive energies (in eV) of the two end-point phases WZ-CdS and RS-AgS. We note that the elastic constants reported in Table 1 are in good agreement with previous experimental and theoretical studies [30, 31]. We see from Table 1 that the elastic constants increase from WZ-CdS to RS-AgS, which means that the material becomes stiffer with increasing silver concentration. In addition, the increase in the cohesive energy suggests that bonds become stronger as Cd decays into Ag. Finally, Figure 6 shows the density of states of WZ-CdS and RS-AgS. We see that throughout the phase transformation the material changes from a semiconductor to a metal. Many properties and behaviors that govern waste form performance are very likely to be impacted by such bonding changes, and must be considered when designing robust waste forms. 


\section{Conclusions}

220 accelerated chemical aging experiments on the ${ }^{109} \mathrm{Cd}_{1-x}{ }^{109} \mathrm{Ag}_{x} \mathrm{~S}$ system. ${ }^{109} \mathrm{Cd}$ decays via electron capture to ${ }^{109} \mathrm{Ag}$ with a half-life of 461 days making it conducive to experimental investigation. ${ }^{109} \mathrm{CdS}$ samples have been prepared and are currently being characterized. To enable more focused analysis of experi-

0 , as expected, the wurtzite phase is the most stable. However, as the concentration of $\mathrm{Ag}$ increases with time, the wurtizite phase becomes less stable than other phases. When all $\mathrm{Cd}$ has decayed to $\mathrm{Ag}$, it is predicted that rocksalt AgS is the most stable phase. At intermediate compositions, it is possible that 230 $h$-MgO. At all compositions greater than $\mathrm{x}=0$, however, phase decomposition is predicted to occur in the thermodynamic limit, suggesting a waste form comprised of many different phases. Potential implications of this phase evolution is briefly surveyed, showing that a large volume change will occur, in addition 235 to variations in elastic constants and bonding.

\section{Acknowledgements}

BD, BPU and CRS acknowledge the support of the U.S. Department of Energy through the LANL LDRD Program. MS acknowledge the support of the Australian Research Council (DP1097076 and FT120100924) and computational resources from National Computational Infrastructure and the iVEC Facility at Murdoch University.

\section{References}

[1] R.A. Wigeland, T.H. Bauer, T.H. Fanning, E.E. Morris, Separations and transmutation criteria to improve utilization of a geologic repository, Nucl. 
[2] Amendment to the record of decision on the Waste Isolation Pilot Plant: Disposal phase supplemental environmental impact statement, Tech. rep., U.S. Dept. of Energy, Office of Environmental Management (2000).

[3] M.E. Long, America's nuclear waste, National Geographic Magazine July (2002) 8 .

[4] W.J. Weber, R.C. Ewing, C.R.A. Catlow, T. D. de la Rubia, L.W. Hobbs, C. Kinoshita, Hj. Matzke, A.T. Motta, M. Nastasi, E.K.H. Salje, E.R. Vance, S.J. Zinkle, Radiation effects in crystalline ceramics for the immobilization of high-level nuclear waste and plutonium, J. Mater. Res. 13 (1998) 1434-84.

[5] R.C. Ewing, W. Lutze, W.J. Weber, Zircon - A host-phase for the disposal of weapons plutonium, J. Mater. Res. 10 (1995) 243-46.

[6] K.E. Sickafus, L. Minervini, R.W. Grimes, J.A. Valdez, M. Ishimaru, F. Li, K.J. McClellan, T. Hartmann, Radiation tolerance of complex oxides, Science 289 (2000) 748-51.

[7] I. Farnan, H. Cho, W.J. Weber, Quantification of actinide alpha-radiation damage in minerals and ceramics, Nature 445 (2007) 190.

[8] K.E. Sickafus, R.W. Grimes, J.A. Valdez, A.R. Cleave, M. Ishimaru, S.M. Corish, C.R. Stanek, B.P. Uberuaga, Radiation-induced amorphization resistance and radiation tolerance in structurally related oxides, Nature Mater. 6 (2007) 217.

[9] C. Jiang, C.R. Stanek, N.A. Marks, K.E. Sickafus, B.P. Uberuaga, Radioparagenesis: The formation of novel compound and crystalline structures via radioactive decay, Philos. Mag. Lett. 90 (2010) 435.

[10] E.R. Vance, R. Roy, J.G. Pepin, D.K. Agrawal, Chemical mitigation of the transmutation problem in crystalline nuclear waste radiophases, J. Mat. Sci. 17 (1982) 947. 
[11] C. Jiang, C.R. Stanek, N.A. Marks, K.E. Sickafus, B.P. Uberuaga, Predicting from first principles the chemical evolution of crystalline compounds due to radioactive decay: The case of the transformation of $\mathrm{CsCl}$ to $\mathrm{BaCl}$, Phys. Rev. B 79 (2009) 132110.

[12] C.R. Stanek, B.P. Uberuaga, B.L. Scott, R.K. Feller, N.A. Marks, Accelerated chemical aging of crystalline nuclear waste forms, Curr. Opinion Sol. State Mater. Sci. 16 (2012) 126.

[13] F. Ulrich, W. Zachariasen, On the crystal structure of alpha and beta CdS, and wurtzite, Z. Kristallogr. 62 (1925) 260.

[14] W.L. Bragg, The crystalline structure of zink oxide, Philos. Mag. 39 (1920) 647.

[15] C.-Y. Yeh, Z.W. Lu, S. Froyen, A. Zunger, Zinc-blende - wurtzite polytypism in semiconductors, Phys. Rev. B 46 (1992) 10086.

[16] D.C. Reynolds, G. Leies, L.L. Antes, R.E. Marburger, Photovoltaic effect in cadmium sulfide, Phys. Rev. 96 (1954) 533.

[17] N. Herron, J.C. Calabrese, W.E. Farneth, Y. Wang, Crystal structure and optical properties of $\mathrm{Cd}_{32} \mathrm{~S}_{14}\left(\mathrm{SC}_{6} \mathrm{H}_{5}\right)_{36} \cdot \mathrm{DMF}_{4}$, A cluster with a 15 angstrom CdS core, Science 259 (1993) 1426.

[18] W. Yu, L. Qu, W. Guo, X. Peng, Experimental determination of the extinction coefficient of CdTe, CdSe, and CdS nanocrystals, Chem. Mater. 15 (2003) 2854.

[19] C.J.M. Rooymans, Structure of the high pressure phase of CdS, CdSe and InSb, Phys. Lett. 4 (1963) 186.

[20] J.A. Corll, Effect of pressure on the elastic parameters and structure of CdS, Phys. Rev. 157 (1967) 623. 
[21] S. Limpijumnong, W.R.L. Lambrecht, Theoretical study of the relative stability of wurtzite and rocksalt phases in $\mathrm{MgO}$ and GaN, Phys. Rev. B 63 (2001) 104103.

[22] D.K. Smith, C.F. Cline, S.B. Austerman, The crystal structure of $\beta$ beryllia, Acta Cryst. 18 (1965) 393.

[23] X. Gonze, J.-M. Beuken, R. Caracas, F. Detraux, M. Fuchs, G.-M. Rignanese, L. Sindic, M.J. Verstraete, G. Zérah, F. Jollet, M. Torrent, A. Roy, M. Mikami, Ph. Ghosez, J.-Y. Raty, D.C. Allan, First-principles computation of material properties: The abinit software project, Comput. Mater. Sci. 25 (2002) 478.

[24] X. Gonze, B. Amadon, P.-M. Anglade, J.-M. Beuken, F. Bottin, P. Boulanger, F. Bruneval, D. Caliste, R. Caracas, M. Cote, T. Deutsch, L. Genovese, Ph. Ghosez, M. Giantomassi, S. Goedecker, D.R. Hamann, P. Hermet, F. Jollet, G. Jomard, S. Leroux, M. Mancini, S. Mazevet, M.J.T. Oliveira, G. Onida, Y. Pouillon, T. Rangel, G.-M. Rignanese, D. Sangalli, R. Shaltaf, M. Torrent, M.J. Verstraete, G. Zerah, J.W. Zwanziger, Abinit: First-principles approach to material and nanosystem properties, Comput. Phys. Commun. 180 (2009) 2582.

[25] P.E. Blöchl, Projector augmented-wave method, Phys. Rev. B 50 (1994) 17953.

[26] J. Korringa, On the calculation of the energy of a Bloch wave in a metal, Physica 13 (1947) 392.

[27] W. Kohn, N. Rostoker, Solution of the Schrödinger equation in periodic lattices with an application to metallic lithium, Phys. Rev 94 (1954) 1111.

[28] A. Zunger, S.-H. Wei, L.G. Ferreira, J.E. Bernard, Special quasirandom structures, Phys. Rev. Lett. 65 (1990) 353. 
[29] C. Jiang, B.P. Uberuaga, K.E. Sickafus, F.M. Nortier, J.J. Kitten, N.A. Marks, C.R. Stanek, Using "radioparagenesis" to design robust nuclear waste forms, Energy and Env. Sci. 3 (2010) 130.

[30] I. Kobiakov, Elastic, piezoelectric and dielectric properties of $\mathrm{ZnO}$ and $\mathrm{CdS}$ single crystals in a wide range of temperatures, Solid State Commun. 35 (1980) 305.

[31] K. Wright, J.D. Gale, Interatomic potentials for the simulation of the zincblende and wurtzite forms of $\mathrm{ZnS}$ and CdS: Bulk structure, properties, and phase stability, Phys. Rev. B 70 (2004) 035211. 